\title{
CACNA1C polymorphism and altered phosphorylation of tau in bipolar disorder
}

\section{Joel Jakobsson, Erik Pålsson, Carl Sellgren, Frida Rydberg, Agneta Ekman, Henrik Zetterberg,} Kaj Blennow and Mikael Landén

\section{Summary}

Several genome-wide association studies and case-control studies have associated the single nucleotide polymorphism (SNP) rs1006737, situated in CACNA1C encoding the alpha 1C subunit of the L-type voltage-gated calcium channel, with bipolar disorder and other psychiatric disorders. However, the causal pathway linking genetic variants in CACNA1C with increased risk for developing brain disorders remains unclear. Here, we explored the association between the rs1006737 SNP and cerebrospinal fluid (CSF) markers. We found a significant association between the risk allele in rs1006737 and a decreased CSF hyperphosphorylated tau/ total tau ratio in patients with bipolar disorder, thus linking variation in the CACNA1C gene to a neurochemical marker of neuroaxonal plasticity in those with this disorder.

\section{Declaration of interest}

None.

\section{Copyright and usage}

(c) The Royal College of Psychiatrists 2016.
Bipolar disorder is a highly heritable psychiatric disorder but the aetiology of the disease is complex, involving multiple genetic and environmental influences. Identifying genetic variants associated with bipolar disorder will increase the understanding of disease mechanisms and may lead to development of targeted therapeutics. Several genome-wide association studies have been conducted that together suggest that the genetic susceptibility of bipolar disorder is clearly polygenic in nature. ${ }^{1}$

One identified single nucleotide polymorphism (SNP) that has been linked to bipolar disorder is of particular interest: rs1006737, which is situated within intron 3 of the gene CACNA1C at chromosome 12, with the $A$ allele associated with an increased risk. ${ }^{1}$ This gene codes the alpha $1 \mathrm{C}$ subunit $\left(\mathrm{Ca}_{\mathrm{v}} 1.2\right)$ of the L-type voltage-gated calcium channel. ${ }^{2} \mathrm{Ca}_{\mathrm{v}} 1.2$ couples transient activation of inward calcium current to transcriptional regulation, which may play a role in dendritic development, neuronal survival, synaptic plasticity, memory formation, learning and behaviour. ${ }^{3}$ The minor $A$ allele has been associated with executive function deficits and has also been coupled to brain areas involved in affective regulation. ${ }^{4}$ This allele has also been associated with schizophrenia and major depression, consistent with shared familial risks across psychiatric disorders. ${ }^{5}$ However, the mechanisms underlying how genetic variants in CACNA1C may modify risk for psychiatric disorders and have an impact on cognition are unclear. One way to increase our understanding of the pathophysiology is to study the neurochemical correlates of CACNA1C gene variants. We have previously characterised the neurochemical profile of bipolar disorder by analyses of cerebrospinal fluid (CSF) markers of neuronal and glial function and degeneration in a large cohort of patients with bipolar disorder and healthy controls. ${ }^{6,7}$ Here, we explored the association between the rs1006737 SNP and these CSF markers in patients with bipolar disorder and healthy controls.

\section{Method}

Our sample comprised 132 patients with bipolar disorder (type I: $n=66$; type II: $n=44$; other types $n=22$ ) and 54 healthy controls. Patients were recruited from the St Göran Bipolar Project, enrolling patients from the bipolar unit at the Northern Stockholm Psychiatric Clinic, Stockholm, Sweden. All patients were assessed using a standardised interview protocol (the Affective Disorders Evaluation $)^{8}$ previously used in the Systematic Treatment Enhancement Program of Bipolar Disorder (STEP-BD). The study was approved by the Regional Ethics Committee in Stockholm and conducted in accordance with the latest Helsinki Protocol. After a complete description of the study, all enrolled patients and controls consented orally and in writing to participate in the study. See the online supplement DS1 for further details.

The CSF sampling (lumbar puncture) was performed when the participants were in a stable euthymic mood. Participants fasted overnight before CSF collection, which took place between 09.00 and $10.00 \mathrm{~h}$. CSF samples were divided into $1.0-1.6 \mathrm{ml}$ aliquots that were stored at $-80^{\circ} \mathrm{C}$ pending analysis. The CSF concentrations of neurofilament light chain (NF-L), S100B, myelin basic protein (MBP) and heart-type fatty acid binding protein (H-FABP), hyperphosphorylated tau (P-tau), total tau (T-tau), soluble amyloid precursor protein alpha (sAPP- $\alpha$ ), soluble amyloid precursor protein beta (sAPP- $\beta$ ), A $\beta 1-42, A \beta X-38$, $\mathrm{A} \beta \mathrm{X}-40$ and $\mathrm{A} \beta \mathrm{X}-42$, were analysed as described by their respective manufacturers. We also included several biomarker ratios in the analysis: $\mathrm{A} \beta \mathrm{X}-42 / \mathrm{A} \beta \mathrm{X}-40, \mathrm{~A} \beta \mathrm{X}-42 / \mathrm{A} \beta \mathrm{X}-38$, and P-tau/T-tau. See online supplement DS1 for further details.

The CACNA1C rs1006737 SNP was genotyped with the KASPar PCR SNP genotyping system (KBioscience, Hoddesdon, UK; www.lgcgenomics.com). SPSS Statistics version 20 was used for all statistical analyses. Analysis of covariance (ANCOVA) with age and gender as covariates was used to analyse effects of rs1006737 on CSF marker concentrations. All $P$-values are presented as two-tailed. Bonferroni correction was used to correct for multiple comparisons $(\alpha=0.05 / 15=0.00333)$.

\section{Results}

The CACNA1C rs1006737 SNP was genotyped in the 132 patients with bipolar disorder. The frequency for the $A$ allele was 0.40 with the genotypes distributed according to Hardy-Weinberg equilibrium $\left(\chi^{2}=0.593, P=0.441\right) . A A(n=23)$ and $A G(n=59)$ genotype carriers were grouped together for analysis of the effects of the $A$ and $G$ alleles on CSF biomarker levels. Demographics of the two groups are displayed in online Table DS1. The groups did not differ significantly with regard to age, gender, smoking status, 
medications, previous episodes of psychosis, Global Assessment of Functioning ${ }^{9}$ score, Clinical Global Investigation ${ }^{10}$ score, diagnosis, duration of illness or number of episodes. A low P-tau/ T-tau ratio was significantly associated with the $A$ allele group $(F(1,128)=13.484, P<0.001, \alpha=0.00333)$ (online Fig. DS1), whereas the rs1006737 genotype had no effect on any of the other biomarkers (online Table DS2). We also found a significant association between the P-tau/T-tau ratio and the $A$ allele under an additive model $(\beta=-0.260, P=0.002$, age and gender as covariates). We next analysed whether this association was specific to bipolar disorder by analysing healthy controls $(n=54)$. The frequency for the $A$ allele of rs1006737 in the control group was 0.30 with the genotypes distributed according to Hardy-Weinberg equilibrium $\left(\chi^{2}=0.675, P=0.411\right)$. In the control group, the rs1006737 SNP was not associated with the P-tau/T-tau ratio $(F(1,50)=0.275$, $P=0.602$ ) or with any of the other CSF biomarkers (online Table DS3).

\section{Discussion}

Variations in CACNA1C has previously been linked to various brain functions but it is unclear how these variations affect the brain on a chemical level. Here, we found a significant association between the rs1006737 SNP and the CSF P-tau/T-tau ratio in patients with bipolar disorder. No association was found in healthy controls, implying that the association is not a general physiological phenomenon but occurs in patients with a psychiatric illness.

$\mathrm{Ca}_{\mathrm{v}} 1.2$ is primarily regulated through an interaction with $\mathrm{Ca}^{2+}$-bound calmodulin $(\mathrm{CaM})$, which also mediates the downstream effects of $\mathrm{Ca}_{\mathrm{v}} 1.2 .{ }^{11}$ Downstream effectors of CaM include the CaMdependent protein kinase (CaMK) cascade and the mitogen-activated protein kinase (MAPK) pathway. ${ }^{3}$ Interestingly, phosphorylation of tau is regulated by a range of proline-directed and nonproline-directed kinases, including CaMK and MAPK, ${ }^{12}$ linking calcium signalling with phosphorylation of tau. Phosphorylation of tau reduces its binding to microtubules leading to destabilisation of microtubules and promoting cytoskeletal flexibility, which have been suggested to be important for axonal and synaptic growth/ development and thus neurodevelopment and synaptic plasticity. ${ }^{12}$ In addition, tau phosphorylation is markedly increased in brain tissue in pathological conditions (i.e. tauopathies), and in CSF in Alzheimer's disease (for a review see Blennow et $a l^{13}$ ). There are, however, no differences between patients with bipolar disorder and controls in either P-tau or T-tau concentrations. ${ }^{7}$ Thus, the difference in P-tau/T-tau between rs1006737 risk allele carriers and non-risk allele carriers probably reflects alterations in the regulation of tau phosphorylation.

Importantly, this study links variations in the CACNA1C gene to neuroaxonal plasticity at the neurochemical level in people with bipolar disorder. Further studies are, however, needed to sort out the biological and clinical significance of altered tau phosphorylation in relation to CACNA1C polymorphism in bipolar disorder and other psychiatric disorders.

\section{Funding}

Financial support was provided through the regional agreement on medical training and clinical research between Stockholm county council and the Karolinska Institutet, and through grants from the Swedish Medical Research Council, Torsten Söderberg's and through grants from the Swedish Medical Research Council, Torsten Söderberg's
Foundation at the Royal Swedish Academy of Sciences, the Brain Foundation and the Swedish Foundation for Strategic Research.

\section{Acknowledgements}

We thank Åsa Källén, Monica Christiansson, Sara Hullberg, Lobna Almasalmeh and Dzemila Secic for excellent technical assistance, the staff at the St Görans Bipolar Affective Disorder Unit, including the coordinator, Martina Wennberg, the study nurse, Agneta Carlswärd-Kjellin, and the data managers, Haydeh Olofsson and Mathias Kardell, for the diagnostic assessments and enrolling patients in this study. Yngve Hallström is acknowledged for performing lumbar punctures on patients and controls.

Joel Jakobsson, PhD, Erik Pålsson, PhD, Institute of Neuroscience and Physiology, Sahlgrenska Academy, University of Gothenburg, Gothenburg, Sweden; Carl Sellgren, PhD, MD, Department of Medical Epidemiology and Biostatistics, Karolinska Institutet, Stockholm, Sweden; Frida Rydberg, MD, Agneta Ekman, PhD, Institute of Neuroscience and Physiology, Sahlgrenska Academy, University of Gothenburg Gothenburg Sweden; Henrik Zetterberg PhD, MD, Institute of Neuroscience and Physiogy, Sahlens of Gothenburg, Gothenburg, Sweden and UCL Institute of Neurology, London, UK; Kaj Blennow, PhD, $\mathrm{MD}$, Institute of Neuroscience and Physiology, Sahlgrenska Academy, University of Gothenburg, Gothenburg, Sweden; Mikael Landén, PhD, MD, Institute of Neuroscience and Physiology, Sahlgrenska Academy, University of Gothenburg, Gothenburg, Department of Medical Epidemiology and Biostatistics, and Department of Clinical Neuroscience, Karolinska Institutet, Stockholm, Sweden

Correspondence: Joel Jakobsson, PhD, Sahlgrenska University Hospital, Blå Stråket 15, Floor 3, SE-413 45 Gothenburg, Sweden. Email: joel.jakobsson@ neuro.gu.se

First received 4 Nov 2014, accepted 27 May 2015

\section{References}

1 Craddock N, Sklar P. Genetics of bipolar disorder. Lancet 2013; 381 1654-62.

2 Catterall WA. Voltage-gated calcium channels. Cold Spring Harb Perspect Biol 2011; 3: a003947.

3 Bhat S, Dao DT, Terrillion CE, Arad M, Smith RJ, Soldatov NM, et al. CACNA1C (Cav1.2) in the pathophysiology of psychiatric disease. Prog Neurobiol 2012; 99: 1-14.

4 Bigos KL, Mattay VS, Callicott JH, Straub RE, Vakkalanka R, Kolachana B, et al. Genetic variation in CACNA1C affects brain circuitries related to mental illness. Arch Gen Psychiatry 2010; 67: 939-45.

5 Smoller JW, Ripke S, Lee PH, Neale B, Nurnberger Jl, Santangelo S, et al. Identification of risk loci with shared effects on five major psychiatric disorders: a genome-wide analysis. Lancet 2013; 381: 1371-9.

6 Jakobsson J, Bjerke M, Ekman CJ, Sellgren C, Johansson AG, Zetterberg $H_{\text {, }}$ et al. Elevated concentrations of neurofilament light chain in the cerebrospinal fluid of bipolar disorder patients. Neuropsychopharmacology 2014; 39: 2349-56.

7 Jakobsson J, Zetterberg $\mathrm{H}$, Blennow K, Ekman CJ, Johansson AG, Landen M. Altered concentrations of amyloid precursor protein metabolites in the cerebrospinal fluid of patients with bipolar disorder. Neuropsychopharmacology 2013; 38: 664-72.

8 Sachs GS, Thase ME, Otto MW, Bauer M, Miklowitz D, Wisniewski SR, et al. Rationale, design, and methods of the systematic treatment enhancement program for bipolar disorder (STEP-BD). Biol Psychiatry 2003; 53: 1028-42.

9 Jones SH, Thornicroft G, Coffey M, Dunn G. A brief mental health outcome scale - reliability and validity of the Global Assessment of Functioning (GAF). Br J Psychiatry 1995; 166: 654-9.

10 Guy W. ECDEU Assessment Manual for Psychopharmacology. Revised DHEW Pub. (ADM). National Institute for Mental Health, 1976.

11 Halling DB, Aracena-Parks P, Hamilton SL. Regulation of voltage-gated Ca2+ channels by calmodulin. SCi STKE 2005; 2005: re15.

12 Avila J, Lucas JJ, Perez M, Hernandez F. Role of tau protein in both physiological and pathological conditions. Physiol Rev 2004; 84: 361-84.

13 Blennow K, Hampel $\mathrm{H}$, Weiner M, Zetterberg $\mathrm{H}$. Cerebrospinal fluid and plasma biomarkers in Alzheimer disease. Nat Rev Neurol 2010; 6: 131-44. 